\title{
The effect of bleaching on tooth enamel
}

\author{
The effect of bleaching on enamel susceptibility to acid erosion and demineralisation
}

\section{A. Pretty, W. M. Edgar and S. M. Higham Br Dent J 2005; 198: 285-290}

\section{Introduction}

The purpose of this in vitro study was to determine if enamel that had been bleached by carbamide (urea) peroxide gel (CPG) was at increased risk of either acid erosion or demineralisation (early caries) than un-bleached enamel.

\section{Methods}

Human incisors were employed. The samples were randomly assigned to one of 4 groups; a) 10\% CPG, b) 16\% CPG, c) $22 \%$ CPG and d) 10\% CPG with xylitol, fluoride and potassium. Each specimen was moistened with saliva and the appropriate formulation placed for 2 hours for a total of 40 hours of exposure. In order to ensure that bleaching had taken place, tooth shades were monitored using the Shade-Eye device. Following the bleaching process, one half of the specimen was subjected to an erosive challenge, the other to a demineralisation system with one half of each sub-sample retained as a non-bleached control. Samples were assessed longitudinally with quantitative light-induced fluorescence (QLF) and at the conclusion of the study with transverse micro-radiography (TMR).

\section{Results}

Erosion was detected in all samples (DQ 126 \pm 23.4 ), in both bleached and non-bleached areas. There was no statistical difference between the bleached and non-bleached areas either within the treatment groups or between them. Caries-like lesions were detected on all samples; TMR revealed sub-surface lesions on all teeth and QLF data supported this (DQ 89 \pm 18.9 ). Following statistical analysis there were no differences detected between the bleached and non-bleached areas, nor between the different concentrations of the bleaching solution.

\section{Conclusion}

These results suggest that tooth bleaching with carbamide (urea) peroxide (using commercially available concentrations) does not increase the susceptibility of enamel to acid erosion or caries.

\section{COMMENT}

Bleaching is quite rightly increasing in popularity in the United Kingdom. It provides a minimally interventive preservative technique for improving the appearance of sound yet discoloured anterior teeth.

Concerns about the safety of bleaching techniques have largely proved unwarranted with the only documented side effect of the procedure being transient sensitivity and gingival irritation when higher concentrations of hydrogen and carbamide peroxide are used.

The authors have investigated whether bleaching with carbamide peroxide gels increases the likelihood of erosion and/or demineralisation post bleaching. This well designed study, albeit an in vitro study, provides us with some reassurance. The authors have clearly demonstrated that bleaching with carbamide peroxide gels of the type tested does not increase enamel susceptibility to subsequent acid erosion and/or demineralisation. In contrast to other studies the writers demonstrate a bleaching effect, which other bleaching studies often omit. Interestingly, the authors demonstrate that increased concentrations of carbamide peroxide above that of the typical 10\% described, do not influence the final bleaching result obtained over a typical treatment period, and they quite rightly point out that higher concentrations merely increase levels of gingival irritation and tooth sensitivity as demonstrated in other studies.

In my opinion, this study provides more evidence to support the safety of bleaching. It is imperative that the difficulties with respect to legal classification of these products are overcome so that patients are not denied a safe minimally interventive procedure to improve the appearance of sound yet discoloured teeth.

\section{P. A. Brunton}

Professor of Restorative Dentistry

Leeds Dental Institute

doi: 10.1038/sj.bdj.4812124

\section{N BRIEF}

- Concentration of carbamide peroxide does not appear to effect bleaching efficacy.

- Bleached teeth, in vitro, are not more susceptible to acid erosion.

- Bleached teeth, in vitro, are not more susceptible to caries.

- These results should prove encouraging to those individuals seeking, and providing bleaching treatments. 